OPEN ACCESS

Edited by:

Angelo Schenone

University of Genoa, Italy

Reviewed by:

Corrado Italo Angelini,

University of Padova, Italy

Raghav Govindarajan,

University of Missouri, United States

*Correspondence:

Margherita Milone

milone.margherita@mayo.edu

Specialty section:

This article was submitted to

Neuromuscular Diseases,

a section of the journal

Frontiers in Neurology

Received: 02 October 2019 Accepted: 26 November 2019 Published: 20 December 2019

Citation:

Pinto MV, Saw J-L and Milone M

(2019) Congenital Vocal Cord

Paralysis and Late-Onset Limb-Girdle

Weakness in MuSK-Congenital

Myasthenic Syndrome.

Front. Neurol. 10:1300

doi: 10.3389/fneur.2019.01300

\section{Congenital Vocal Cord Paralysis and Late-Onset Limb-Girdle Weakness in MuSK-Congenital Myasthenic Syndrome}

\author{
Marcus V. Pinto, Jacqui-Lyn Saw and Margherita Milone* \\ Department of Neurology, Mayo Clinic, Rochester, MN, United States
}

A 30-year-old woman with congenital vocal cord paralysis presented for evaluation of fatigable proximal upper limb weakness and difficulty maintaining the neck erect. Neurologic examination showed bilateral asymmetric eyelid ptosis, mild weakness (MRC $4 / 5)$, and atrophy of neck extensors and shoulder girdle muscles, whereas lower limb muscle strength was normal. Repetitive nerve stimulation revealed decremental responses in orbicularis oculis and trapezius. Needle electromyography demonstrated myopathic changes in proximal and paraspinal muscles. Acetylcholine receptor and muscle skeletal receptor tyrosine kinase (MuSK) antibodies, creatine kinase (CK), and lactate were negative or normal. Next-generation sequencing detected two heterozygous variants in the MUSK gene. One variant, c. $79+2 T>G$, is a known pathogenic variant, and the other, c.2165T>C (p.V722A), is a novel missense variant, predicted to be pathogenic by in silico analysis. The two variants were proven to be in trans. This case expands the clinical and molecular spectrum of MuSK congenital myasthenic syndromes.

\footnotetext{
Keywords: congenital myasthenic syndrome, muscle skeletal receptor tyrosine kinase, limb girdle, vocal cord paralysis, CMS, MuSK, Neuromuscular Junction disorder
}

\section{INTRODUCTION}

Congenital myasthenic syndromes (CMS) are a heterogeneous group of hereditary neuromuscular disorders due to defects of the neuromuscular transmission. CMSs are characterized by fatigable muscle weakness, ptosis, and ophthalmoparesis and often manifest in infancy or childhood (1). However, a fair number of CMS patients remain undiagnosed until adulthood. Mutations in at least 30 genes encoding for proteins involved in the neuromuscular transmission have been linked to CMS (2). Muscle skeletal receptor tyrosine kinase (MuSK) CMS is a rare autosomal recessive CMS, and only 22 cases have been reported worldwide (3-13). MuSK is a post-synaptic transmembrane protein consisting of three immunoglobulin-like domains, a frizzled cysteine-rich domain, and a protein kinase domain. It has a crucial role in the formation and stabilization of the neuromuscular junction (14), and its disruption in mice has been shown to result in failure to form neuromuscular junctions and death (15). MuSK deficiency usually presents at birth with eyelid ptosis and/or respiratory distress, followed by ocular, facial, and proximal limb 
muscle weakness (1). However, it can a wider phenotypic spectrum that includes fetal akinesia deformation sequence syndrome (16), congenital vocal cord paralysis (CVCP) (13), or late-onset limb-girdle weakness (12). CVCP was previously reported as presenting symptom of Dok7 CMS, rare cases of fast-channel syndrome, and later in MuSK deficiency CMS (1, 9, 13). However, all patients with MuSK CMS and CVCP at disease-onset developed weakness in childhood or presented with isolated CVCP $(9,10,12,13)$. Herein, we describe a patient with MuSK CMS manifesting with CVCP and late-onset limbgirdle weakness.

\section{CASE REPORT}

The patient is a 30-year-old woman who was born at term to non-consanguineous parents. Immediately after uncomplicated delivery, she developed respiratory distress and required intubation. She received a diagnosis of bilateral CVCP. She was discharged home with a tracheostomy without ventilator support or oxygen. She had no episodes of apnea. She underwent bilateral arytenoidectomy was successfully decannulated at age 3 . In the mean time, she achieved normal motor milestones and kept up with her peers in gymnastics. She had, however, some exertional dyspnea and stridor. Mild eyelid ptosis was noted in childhood. During pregnancy, 15 months before presenting to our institution, she noted fatigable weakness of proximal upper limb and neck extensors muscles as well as shoulder girdle muscle atrophy. She had no diplopia, dysphagia, lower limb weakness, or exacerbation of the respiratory symptoms. She had no myalgia. Her 11-month-old daughter is well and has met motor development on time. Her 33-year-old brother and parents, of English and German descent, are asymptomatic. Neurological examination showed bilateral asymmetric eyelid ptosis (Figures 1A,B), slight ophthalmoparesis without diplopia, moderate lower facial weakness, mild tongue weakness, and mildly reduced elevation of the soft palate. Neck extensors and shoulder girdle muscles were mild weak and atrophic [Medical Research Council (MRC) 4/5], whereas lower limb muscle strength was normal. Deltoid fatigability was observed. She had mild high arched palate, micrognathia, long neck, bilateral scapular winging (Figures 1C,D), and hyperextensibility of the interphalangeal and metacarpophalangeal joints. Repetitive nerve stimulation at $2 \mathrm{~Hz}$ revealed decremental responses in orbicularis oculis $(-7 \%$ at rest and $-15 \%$ post-exertion) and trapezius $(-22 \%$ at rest and $-35 \%$ post-exertion). The more pronounced decrement in the trapezius, compared with that recorded in the orbicularis oculi, reflected the clinically more prominent involvement of the shoulder girdle muscles compared with the relatively spared orbicularis oculi. There was no facilitation. Needle electromyography demonstrated myopathic changes without abnormal spontaneous muscle activity in proximal and paraspinal muscles. Acetylcholine receptor and MuSK antibodies, creatine kinase (CK), and

Abbreviations: CMS, congenital myasthenic syndromes; CVCP, congenital vocal cord paralysis; MuSK, muscle skeletal receptor tyrosine kinase; MUSK, muscle skeletal receptor tyrosine kinase gene. lactate were negative or normal. Pulmonary function tests showed reduced maximal respiratory pressures with features suggestive of fixed central airway obstruction. Next-generation sequencing detected two heterozygous variants in the MUSK gene. One variant, c.79+2T $>\mathrm{G}$, affects a donor splice site in intron 1 and is expected to result in absent or disrupted protein product. This variant was previously reported in two MuSK CMS patients with CVCP (9). The second variant, c. $2165 \mathrm{~T}>\mathrm{C}$ (p.V722A), is a novel missense variant that affects a highly conserved amino acid, not present in the population databases and predicted to be deleterious by SIFT and PolyPhen-2 P2. The two variants were proven to be in trans $(\mathrm{C} .79+2 \mathrm{~T}>\mathrm{G}$ from mother and c.2165T $>\mathrm{C}$ from father). Albuterol $4 \mathrm{mg}$ orally resulted in normal strength of the neck flexors, neck extensors, biceps brachii, and triceps bilaterally.

\section{DISCUSSION}

This patient expands the clinical and molecular spectrum of MuSK CMS, a rare form of neuromuscular junction defect. Indeed, she presented with adult-onset limb-girdle weakness, in addition to CVCP, and compound heterozygosity for a




novel missense MUSK variant. The novel p.V722A variant is located in the tyrosine kinase region of MuSK and very close to a previously reported pathogenic variant (p.A727V) (12). Owen et al. described four MuSK CMS patients with lateonset limb-girdle predominant weakness and eyelid ptosis who were diagnosed in adulthood, but none of them had CVCP. These adult patients carried compound heterozygous MUSK variants affecting exclusively the protein kinase region, leading the authors to hypothesize that mutations in this region may be specific for late-onset limb-girdle weakness (12). However, recently, isolated CVCP was reported in two children (siblings) with compound heterozygous variants in the tyrosine kinase domain of MuSK (p.A763T and p.R816X) (13). Dok7 CMS is one of the most common CMS diagnosed in adulthood and often results in a mild phenotype with limb-girdle predominant weakness (2), as observed in our patient, mimicking a limbgirdle muscular dystrophy. It is unknown why our patient, like other CMS patients $(1,2)$, had initial symptoms very early in life and then partially improved but developed limbgirdle weakness decades later. Similarly to MuSK CMS, also Dok7 CMS can cause CVCP and usually spares extra-ocular muscles $(1,5,12,13)$. The phenotypic overlapping between these two forms of CMS could stem from a similar underlying mechanism, secondary to the close interplay between MuSK and Dok7. MuSK is activated extracellularly by the neural isoform of agrin through binding LDL receptor related protein 4 (LRP4) and intracellularly by $\operatorname{Dok} 7(1,5,15)$. Dok7 recruits MuSK through phosphorylations of its Tyr553 residue, which then leads to phosphorylation of the acetylcholine receptor (AChR) $\beta$-subunit, AChR clustering, and reorganization of the actin cytoskeleton $(1,5)$. Severe impairment of the interaction between

\section{REFERENCES}

1. Engel AG, Shen XM, Selcen D, Sine SM. Congenital myasthenic syndromes: pathogenesis, diagnosis, and treatment. Lancet Neurol. (2015) 14:420-34. doi: 10.1016/S1474-4422(14) 70201-7

2. Kao JC, Milone M, Selcen D, Shen XM, Engel AG, Liewluck T. Congenital myasthenic syndromes in adult neurology clinic: a long road to diagnosis and therapy. Neurology. (2018) 91:e1770-7. doi: 10.1212/WNL.00000000000 06478

3. Chevessier F, Faraut B, Ravel-Chapuis A, Richard P, Gaudon K, Bauche S, et al. MUSK, a new target for mutations causing congenital myasthenic syndrome. Hum Mol Genet. (2004) 13:3229-40. doi: 10.1093/hmg/ ddh 333

4. Mihaylova V, Salih MA, Mukhtar MM, Abuzeid HA, El-Sadig SM, von der Hagen, M, et al. Refinement of the clinical phenotype in muskrelated congenital myasthenic syndromes. Neurology. (2009) 73:19268. doi: 10.1212/WNL.0b013e3181c3fce9

5. Maselli RA, Arredondo J, Cagney O, Ng JJ, Anderson JA, Williams C, et al. Mutations in MUSK causing congenital myasthenic syndrome impair MuSK-Dok-7 interaction. Hum Mol Genet. (2010) 19:23709. doi: $10.1093 / \mathrm{hmg} / \mathrm{ddq} 110$

6. Maggi L, Brugnoni R, Scaioli V, Winden TL, Morandi L, Engel AG, et al. Marked phenotypic variability in two siblings with congenital myasthenic syndrome due to mutations in MUSK. J Neurol. (2013) 260:28946. doi: 10.1007/s00415-013-7118-5
MuSK and Dok7, resulting in abnormal endplate formation and presynaptic differentiation, was demonstrated in a compound heterozygous MuSK CMS patient (5). Mice lacking MuSK do not develop neuromuscular junctions and die at birth from respiratory failure, confirming the vital role of the MuSK signaling pathways (15).

Our patient, similarly to most MuSK CMS patients (12), responded to albuterol, which should be the first-line therapy in MuSK CMS and other CMS featured by impairment of the agrinLRP4-MuSK-Dok7 pathway. Acetylcholinesterase inhibitors may worsen weakness in MuSK CMS, as in MuSK-antibody positive myasthenia gravis, and should be avoided.

\section{ETHICS STATEMENT}

Written informed consent was obtained from the participant for the publication of this case report and all identifiable data and images.

\section{AUTHOR CONTRIBUTIONS}

MP and MM performed data collection, drafting of final manuscript, approval, and critical review of final form. J-LS performed data collection, approval, and critical review of final form.

\section{ACKNOWLEDGMENTS}

We thank the patient for agreeing to the publication of her photographs for scientific and educational purpose. MM receives research support from a Mayo Clinic benefactor.

7. Ben Ammar A, Soltanzadeh P, Bauche S, Richard P, Goillot E, Herbst R, et al. A mutation causes MuSK reduced sensitivity to agrin and congenital myasthenia. PLoS ONE. (2013) 8:e53826. doi: 10.1371/journal.pone.00 53826

8. Gallenmuller C, Muller-Felber W, Dusl M, Stucka R, Guergueltcheva V, Blaschek A, et al. Salbutamol-responsive limb-girdle congenital myasthenic syndrome due to a novel missense mutation and heteroallelic deletion in MUSK. Neuromuscul Disord. (2014) 24:31-5. doi: 10.1016/j.nmd.2013. 08.002

9. Al-Shahoumi R, Brady LI, Schwartzentruber J, Tarnopolsky MA. Two cases of congenital myasthenic syndrome with vocal cord paralysis. Neurology. (2015) 84:1281-2. doi: 10.1212/WNL.0000000000001396

10. Giarrana ML, Joset P, Sticht H, Robb S, Steindl K, Rauch A, et al. A severe congenital myasthenic syndrome with "dropped head" caused by novel MUSK mutations. Muscle Nerve. (2015) 52:668-73. doi: 10.1002/mus. 24687

11. Luan X, Tian W, Cao L. Limb-girdle congenital myasthenic syndrome in a Chinese family with novel mutations in MUSK gene and literature review. Clin Neurol Neurosur. (2016) 150:41-45. doi: 10.1016/j.clineuro.2016. 08.021

12. Owen D, Topf A, Preethish-Kumar V, Lorenzoni PJ, Vroling B, Scola RH, et al. Recessive variants of MuSK are associated with late onset CMS and predominant limb girdle weakness. Am J Med Genet. Part A. (2018) 176:1594601. doi: 10.1002/ajmg.a.38707

13. Murali C, Li D, Grand K, Hakonarson H, Bhoj E. Isolated vocal cord paralysis in two siblings with compound heterozygous variants in MUSK: 
expanding the phenotypic spectrum. Am J Med Genet. Part A. (2019) 179:6558. doi: 10.1002/ajmg.a.61060

14. Hubbard SR, Gnanasambandan K. Structure and activation of MuSK, a receptor tyrosine kinase central to neuromuscular junction formation. Biochim Biophys Acta. (2013) 1834:2166-9. doi: 10.1016/j.bbapap.2013. 02.034

15. DeChiara TM, Bowen DC, Valenzuela DM, Simmons MV, Poueymirou WT, Thomas S, et al. The receptor tyrosine kinase MuSK is required for neuromuscular junction formation in vivo. Cell. (1996) 85:50112. doi: $10.1016 / \mathrm{S} 0092-8674(00) 81251-9$

16. Wilbe M, Ekvall S, Eurenius K, Ericson K, Casar-Borota O, Klar J, et al. MuSK: a new target for lethal fetal akinesia deformation sequence (FADS). J Med Genet. (2015) 52:195-202. doi: 10.1136/jmedgenet-2014-102730
Conflict of Interest: MM receives compensation to serve as associate editor of Neurology Genetics.

The remaining authors declare that the research was conducted in the absence of any commercial or financial relationships that could be construed as a potential conflict of interest.

Copyright (c) 2019 Pinto, Saw and Milone. This is an open-access article distributed under the terms of the Creative Commons Attribution License (CC BY). The use, distribution or reproduction in other forums is permitted, provided the original author(s) and the copyright owner(s) are credited and that the original publication in this journal is cited, in accordance with accepted academic practice. No use, distribution or reproduction is permitted which does not comply with these terms. 